\title{
STEREO and ACE Observations of CIR Particles
}

\author{
R. A. Leske*, R. A. Mewaldt*, G. M. Mason, C. M. S. Cohen*, \\ A. C. Cummings* , A. J. Davis*, A. W. Labrador*, H. Miyasaka*, \\ E. C. Stone ${ }^{*}$, M. E. Wiedenbeck ${ }^{* *}$ and T. T. von Rosenvinge ${ }^{\ddagger}$ \\ ${ }^{*}$ California Institute of Technology, Pasadena, CA 91125 USA \\ $\dagger$ Johns Hopkins University/Applied Physics Laboratory, Laurel, MD 20723 USA \\ ** Jet Propulsion Laboratory, California Institute of Technology, Pasadena, CA 91109 USA \\ ${ }^{\ddagger}$ NASA/Goddard Space Flight Center, Greenbelt, MD 20771 USA
}

\begin{abstract}
In the present solar minimum, corotating interaction regions (CIRs) produce frequent particle enhancements at $1 \mathrm{AU}$ as observed at STEREO and ACE. As the two STEREO spacecraft move apart, differences in CIR time profiles observed at each spacecraft are becoming large. The timing differences are often roughly similar to the corotation time lag between the two spacecraft, however many of the features seen at Ahead and Behind require more than just a time shift. Perhaps transient disturbances in the solar wind affect connection to or transport from the shock, or temporal changes occur in the CIR shock itself. Additional timing differences of $>1$ day result from the different heliographic latitudes of the two STEREO spacecraft.
\end{abstract}

Keywords: Interplanetary physics, Corotating streams, Energetic particles, STEREO, ACE PACS: 96.50.-e,96.50.Qx, 96.50.Vg

\section{INTRODUCTION}

Corotating interaction regions (CIRs) develop when coronal hole high-speed solar wind interacts with the preceding slow solar wind. Forward and reverse shocks often form at CIR boundaries, usually beyond $1 \mathrm{AU}$, and accelerate particles to $\sim 20 \mathrm{MeV} / \mathrm{nucleon}$. CIRs are especially prominent during the declining phase of the solar cycle, when the heliospheric magnetic field has a well-developed sector structure and coronal holes may extend to low latitudes. Additional CIR properties and their effects on energetic particles are discussed in various reviews (e.g., [1]).

Among the instruments making up the IMPACT suite on the twin STEREO spacecraft are the Low Energy Telescope (LET) [2] and Suprathermal Ion Telescope (SIT) [3], which measure elemental composition from $\mathrm{H}$ to beyond $\mathrm{Ni}$ at $\sim 2$ to $\sim 50 \mathrm{MeV} /$ nucleon and $\sim 0.05$ to $5 \mathrm{MeV} /$ nucleon respectively. We report CIR observations from these instruments and from ACE during 2007 and extending through early 2008.

\section{SPECTRA AND COMPOSITION OBSERVATIONS}

Except for the large solar energetic particle (SEP) events in December 2006 and two small events in May 2007, the Sun has been very quiet in terms of energetic particle production since the STEREO launch in October 2006. Nearly all the low energy protons

\footnotetext{
CP1039, Particle Acceleration and Transport in the Heliosphere and Beyond $7^{\text {th }}$ Annual Astrophysics Conference edited by G. Li, Q. Hu, O. Verkhoglyadova, G. P. Zank, R. P. Lin, and J. Luhmann (1) 2008 American Institute of Physics 978-0-7354-0566-0/08/\$23.00
} 


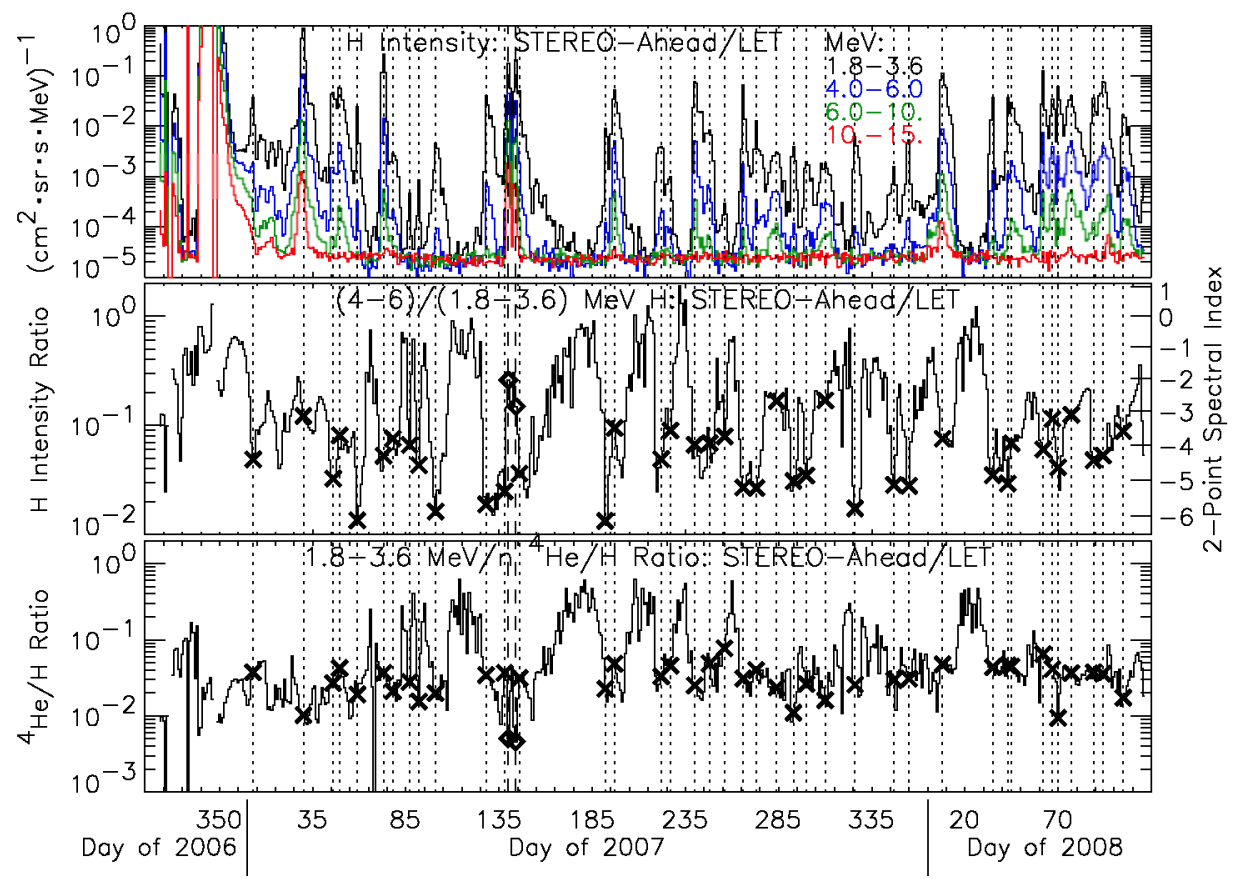

FIGURE 1. Daily-averaged proton intensities from STEREO/LET on the Ahead spacecraft (top panel) at the energies indicated. Dotted lines mark the local maxima of the CIR events; dashed lines indicate the May 2007 SEP events. The middle panel shows the ratio of the (4-6)/(1.8-3.6) MeV proton intensities, with the corresponding power-law spectral index shown on the right axis. Values at the CIR peaks are indicated by crosses and at the SEP peaks by diamonds. The bottom panel shows the $\mathrm{He} / \mathrm{H}$ ratio at 1.8-3.6 $\mathrm{MeV} /$ nucleon, with the CIR and SEP peaks indicated as before.

detected by STEREO/LET since the start of 2007 (Fig. 1) have been due to CIRs, providing an extended period in which to study CIR particles uncontaminated by SEPs.

As Fig. 1 shows, the CIR proton spectra are quite steep at energies of several MeV, with spectral indices ranging from $\sim-3$ to as soft as $\sim-6$. A similar range of spectral indices at a comparable energy (0.91-3.6 MeV/nucleon) was found for He spectra in the recent CIR heavy ion survey of Mason et al. $[4,5]$. Note that the two May 2007 SEP events had considerably harder spectra, especially compared with the CIRs immediately preceeding and following them. The days with the hardest spectra in Fig. 1 are those with the lowest proton intensities, corresponding to the galactic cosmic ray (GCR) and anomalous cosmic ray (ACR) quiet-time levels.

The $\mathrm{He} / \mathrm{H}$ ratio at 1.8-3.6 MeV/nucleon in these events is also shown in Fig. 1. In CIRs He/H varies by a factor of $\sim 2$ on either side of $\sim 0.03$. The May 2007 SEP events have significantly lower He/H ratios of $\sim 0.005$. The highest He/H ratios appear during the quietest days and are due to the presence of ACR He at these energies. Our CIR He/H ratio is similar to that reported in earlier measurements at comparable energies [6].

$\mathrm{H}$ and He spectra in 6 CIR events when ACE and STEREO were still close together 


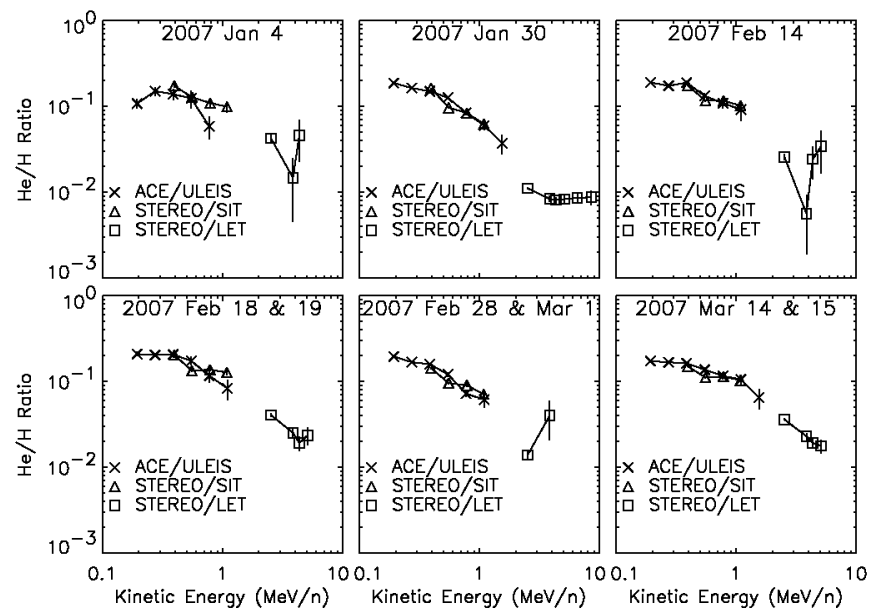

FIGURE 2. The He/H ratio as a function of energy in 6 CIR events, using data from ULEIS on $\mathrm{ACE}$ (crosses) and from SIT (triangles) and LET (squares) on STEREO-Ahead.

in early 2007 , covering energies from $\sim 0.1$ to $>10 \mathrm{MeV} /$ nucleon, are presented and discussed in [7]; the energy dependence of the $\mathrm{He} / \mathrm{H}$ ratio in these events is shown here in Fig. 2. Although heavier ion abundance ratios have been found to be nearly independent of energy over this same energy range [4,5], all 6 of these events show a significant energy dependence in the He/H ratio.

\section{TIMING OBSERVATIONS AND DISCUSSION}

LET CIR measurements at the two STEREO spacecraft are compared with each other and with solar wind speed and magnetic field direction in Fig. 3. As expected, each particle increase is associated with a high-speed stream. However, the converse is not true; often during this period a high-speed stream generates no detectable particle increase above a few MeV [7]. Sometimes a second, smaller particle increase is seen when the solar wind velocity is declining (e.g., panels 1-3 and 5); these generally have a harder spectrum (Fig. 1) than the initial event, and are probably due to connection to the reverse shock [8]. At times, rather than causing a particle increase, a high-speed stream is associated with an abrupt end to the particle event (e.g., panels 9, 10, and 15), presumably because the connection to the CIR shock is broken (but it is not obvious why the second stream does not also form a shock and accelerate particles).

Early in the mission the spacecraft were close together (Fig. 4) and the LET Ahead and Behind proton time profiles were nearly identical, but differences in both the timing and intensity are becoming greater as the spacecraft move farther apart. Closer examination [7] shows that CIR onsets early in the mission happened first at Ahead, sometimes by nearly 2 hours before appearing at Behind; since mid-2007 Behind is always first, sometimes by $\sim 3$ days. To first order this is as expected. Early in 2007 the spacecraft 

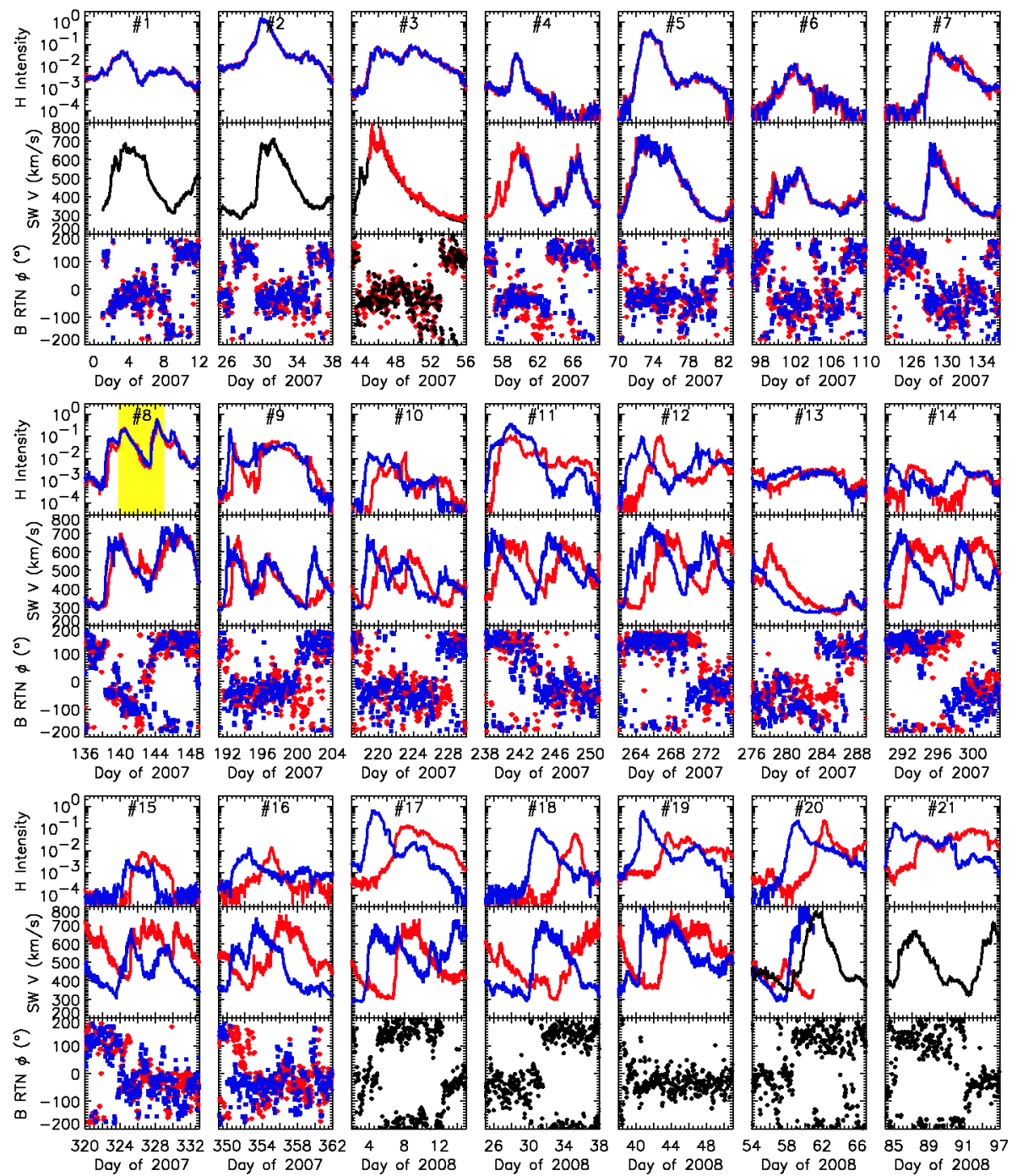

FIGURE 3. Data from STEREO/Ahead (red), STEREO/Behind (blue), and ACE (black, where STEREO data are unavailable) during 21 CIR periods. In each triple panel, the top shows 1.8-3.6 $\mathrm{MeV}$ protons from STEREO/LET, the middle shows solar wind velocity from STEREO/PLASTIC or ACE/SWEPAM, and the bottom shows magnetic longitude from MAG on STEREO or ACE. The yellow shaded region in panel \#8 indicates when the protons were primarily from SEPs rather than CIRs.

were more nearly radially-aligned than the typical $45^{\circ}$ magnetic field angle (Fig. 4), so a corotating field line at a typical angle would contact Ahead first (by a small amount, since the spacecraft were not far apart). Later, as the Ahead-Behind-Sun angle exceeded that usually seen for the magnetic field, a corotating field line connected to the CIR 

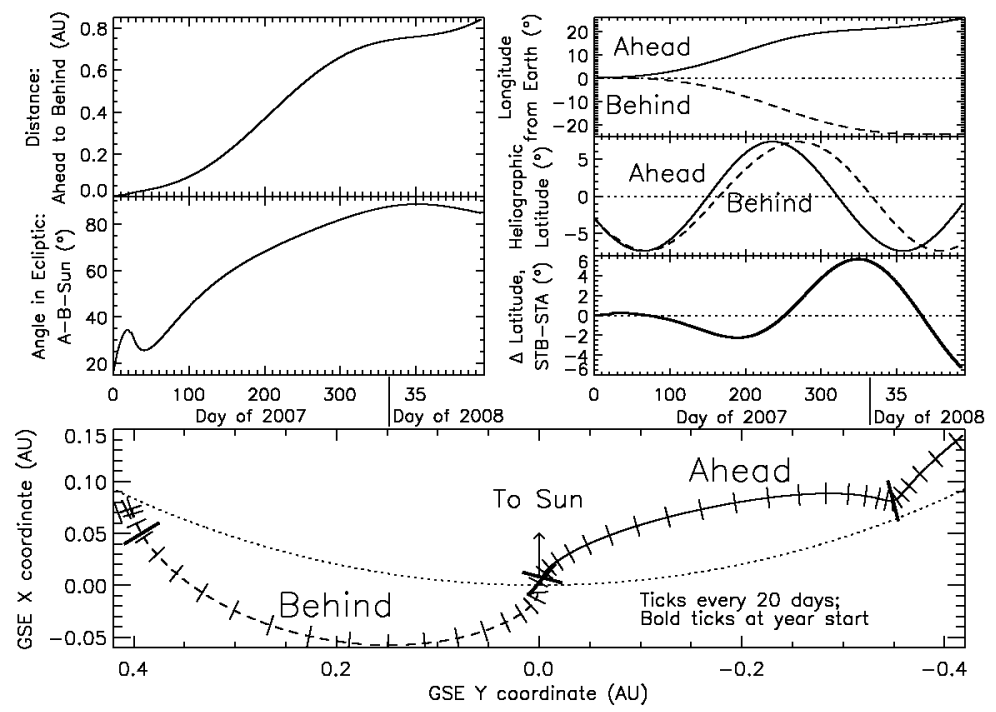

FIGURE 4. STEREO position data from the beginning of 2007 to the present, with quantities as indicated. Where separate curves for each spacecraft are used, Ahead is shown as a solid line and Behind as a dashed line. The dotted arc in the bottom panel indicates a Sun-centered circle of $1 \mathrm{AU}$ radius.

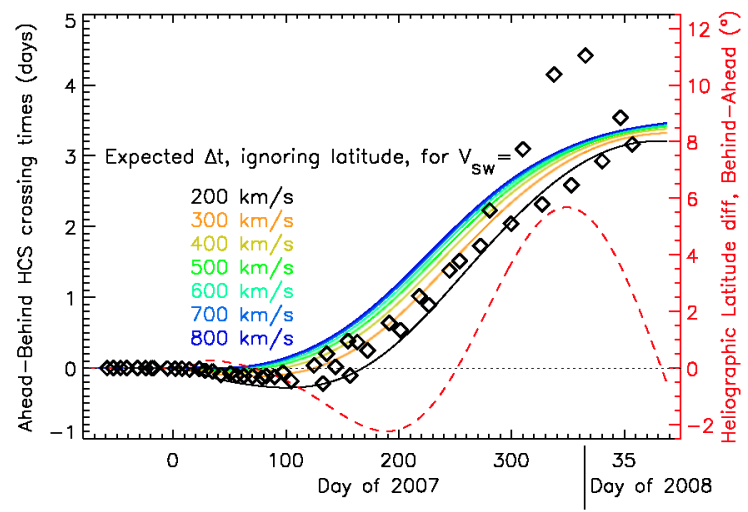

FIGURE 5. Calculated corotation times between the STEREO spacecraft (based on their heliographic radii and longitudinal separation) for a variety of solar wind speeds (colored curves) compared with crossing time differences of the HCS (diamonds), which depend on the HCS tilt at the spacecraft latitude. The difference in latitude of the two spacecraft is also shown (dashed line; right axis).

source would sweep past Behind first; by early 2008 with a longitudinal separation of $50^{\circ}$, the corotation time between the two spacecraft was 3.8 days. However, the time differences are not growing monotonically. One of the most obvious examples is that the difference in panel 15 of Fig. 3 is significantly less than that in panel 12; other more subtle cases also exist. In addition, timing differences between Behind and Ahead at the 
start of an event can be quite different from those at the end of the same event (e.g., panels 10 and 15). In some cases these effects may be due to changes in the magnetic field directions, but in others this does not account for the timing differences [9].

Many of the CIR increases happen at or very near a magnetic sector crossing (Fig. 3), consistent with a study that found $\sim 70 \%$ of all clean heliospheric current sheet (HCS) crossings at 1 AU occurred within 1 day before a high-speed solar wind stream [10]. Even if the boundary of the CIR region (or region connected to a polar coronal hole) is not precisely a sector boundary but at least roughly parallel to it, a small difference in heliographic latitude can make a rather large difference in the boundary crossing time. Although the STEREO spacecraft orbit essentially in the ecliptic plane, their large angular separation along with the $\sim 7^{\circ}$ tilt of the solar rotation axis relative to the ecliptic means that their heliographic latitudes are quite different at times, up to nearly $6^{\circ}$ so far (Fig. 4). If the boundary of the CIR region is tilted by $30^{\circ}$ to the solar equator, for example, a $6^{\circ}$ latitude difference corresponds to $\sim 10^{\circ}$ of longitude, or 0.8 day difference in the boundary crossing time due solely to this small latitude difference.

To illustrate in more detail, we have taken the location of the HCS provided by the Wilcox Solar Observatory (using their "classic" model with the source surface at 2.5 $\mathrm{R}_{s}$ ), convected it out to each STEREO orbit at a constant $300 \mathrm{~km} / \mathrm{s}$, and determined the HCS crossing time for each STEREO spacecraft (Fig. 5). The difference in crossing times between the two spacecraft differ from those expected for simple corotation (that is, ignoring the latitude differences) by more than a day when the latitude differences are large. Effects of similar or larger magnitude would be expected for polar coronal hole boundary crossings at $1 \mathrm{AU}$.

Several of the CIRs in mid-2007 were also observed on Ulysses during its third fast latitude scan, at very different latitudes, longitudes, and radii than STEREO or ACE. In future work we will compare our data with the Ulysses measurements to further test ideas of the effect of CIR geometry on the timing differences.

\section{ACKNOWLEDGMENTS}

This work was supported by NASA grants NAS5-03131 and NAG5-12929. We thank STEREO/PLASTIC (NASA contract NAS5-00132) and MAG investigators for making their data publicly available, and the Wilcox Solar Observatory for the HCS data.

\section{REFERENCES}

1. I. G. Richardson, Space Sci. Rev. 111, 267 (2004).

2. R. A. Mewaldt, et al., Space Sci. Rev. (2007), doi:10.1007/s11214-007-9288-x.

3. G. M. Mason, et al., Space Sci. Rev. (2007), doi:10.1007/s11214-006-9087-9.

4. G. M. Mason, et al., Astrophys. J. 678, 1458 (2008).

5. G. M. Mason, et al., AIP Conf. Proc. (2008), this volume.

6. I. G. Richardson, et al., J. Geophys. Res. 98, 13 (1993).

7. R. A. Leske, et al., Proc. 30th Internat. Cosmic Ray Conf. (Merida) (2008), in press.

8. D. Lario, and E. C. Roelof, J. Geophys. Res. 112, A09107 (2007), doi:10.1029/2007JA012414.

9. R. Müller-Mellin, et al., Proc. 30th Internat. Cosmic Ray Conf. (Merida) (2008), in press.

10. G. Borrini, et al., J. Geophys. Res. 86, 4565 (1981). 